This item was submitted to Loughborough's Research Repository by the author.

Items in Figshare are protected by copyright, with all rights reserved, unless otherwise indicated.

\title{
Superfast ion scattering by solar wind discontinuities
}

PLEASE CITE THE PUBLISHED VERSION

https://doi.org/10.1103/PhysRevE.102.033201

PUBLISHER

American Physical Society

VERSION

AM (Accepted Manuscript)

PUBLISHER STATEMENT

This paper was accepted for publication in the journal Physical Review E: covering statistical, nonlinear, biological, and soft matter physics and the definitive published version is available at https://doi.org/10.1103/PhysRevE.102.033201.

\section{LICENCE}

CC BY-NC-ND 4.0

\section{REPOSITORY RECORD}

Artemyev, Anton, Anatoly Neishtadt, Alexey Vasiliev, Vassilis Angelopoulos, Alexander Vinogradov, and Lev Zelenyi. 2020. "Superfast lon Scattering by Solar Wind Discontinuities". Loughborough University. https://hdl.handle.net/2134/12837506.v1. 


\title{
Superfast ion scattering by solar wind discontinuities
}

\author{
Artemyev A.V. ${ }^{1,2}{ }^{*}$ Neishtadt A.I. ${ }^{2,3}$, Vasiliev A.A. ${ }^{2}$, Angelopoulos V. ${ }^{1}$, Vinogradov A.A. ${ }^{2}$, and Zelenyi L.M. ${ }^{2,4}$ \\ 1 Institute of Geophysics and Planetary Physics, University of California, \\ Los Angeles, CA, USA; ${ }^{2}$ Space Research Institute RAS, \\ Moscow, Russia; ${ }^{3}$ Department of Mathematical Sciences, \\ Loughborough University, Loughborough LE11 3TU, UK; \\ ${ }^{4}$ Moscow Institute of Physics and Technology (State University), 141700, Dolgoprudnyi, Moscow oblast, Russia
}

(Dated: August 20, 2020)

\begin{abstract}
Large-amplitude fluctuations of the solar wind magnetic field can scatter energetic ions. One of the main contributions to these fluctuations is provided by solar wind discontinuities, i.e. rapid rotations of the magnetic field. This study shows that the internal configuration of such discontinuities plays a crucial role in energetic ion scattering in pitch-angles. Kinetic-scale discontinuities accomplish very fast ion pitch-angle scattering. The main mechanism of such pitch-angle scattering is the adiabatic invariant destruction due to separatrix crossings in the phase space. We demonstrate that efficiency of this scattering does not depend on the magnetic field component across the discontinuity surface, i.e. both rotational and almost tangential discontinuities scatter energetic ions with the same efficiency. We also examine how the strong scattering effect depends on the deviations of the discontinuity magnetic field from the force-free one.
\end{abstract}

The supersonic solar wind is filled by large-scale magnetic field fluctuations, such as Alfven waves and solar wind discontinuities (SWD) $[1,2]$. Recent Parker Solar Probe observations found an abundance of such SWD at small radial distances from the Sun $[3,4]$. These fluctuations contribute significantly to the solar wind heating [5], as well as acceleration [6] and scattering of energetic ions $[7,8]$. Such scattering results in random jumps of ion pitch-angles due to the ion interaction with magnetic field fluctuations [9], which are dominated by magnetic field rotations $[10,11]$.

Energetic ion ( $>$ few keVs of thermal energy) scattering is responsible for the cross-field transport [12] affecting ion propagation time in the heliosphere [13] and ion spatial distributions [14]. The classical theory of ion scattering is based on consideration of an ensemble of random magnetic field fluctuations $[7,8,15]$, whereas the internal structure of such fluctuations has not been studied in detail. Compressional fluctuations, e.g. high- $\beta$ current sheets, are known to be effective ion scatters [16]. However, solar wind fluctuations are dominated by compressionless magnetic field rotations (so-called rotational SWD, see $[1,10]$ ), and there is no theory describing the role of the SWD internal structure in the ion scattering. This study focuses on the ion scattering by such SWD consistent with observed magnetic field configurations.

Figure 1 shows a typical example of rotational SWDs observed by the ARTEMIS spacecraft [17] at 1 AU. The magnetic field rotates while $|\mathbf{B}| \approx$ const (see panel (a)): the reversal of the magnetic field component along the maximum variance direction, $B_{l}$ is compensated by a peak of the absolute value of the intermediate variance direction component, $B_{m}$ (see panel (b)). Using the solar wind velocity projected onto the normal direction to the SWD surface, that of the minimum variance, $\mathbf{n}$, [e.g., 18], we transform time to space $r_{n}$ and normalize it to the proton inertial length $d_{p}=\sqrt{m_{p} c^{2} / 4 \pi n_{p} e^{2}}$ where $n_{p}$ is the solar wind density. The spatial scale of this SWD is few $d_{p}$, and the current density peak reaches $j_{m} \approx(c / 4 \pi) \partial B_{l} / \partial r_{n} \sim 30 \mathrm{nA} / \mathrm{m}^{2}$ (panel (c)). These are typical scale and $j_{m}$ for the most intense SWDs with $B_{l}$ magnitude $B_{0}$ comparable to the $|\mathbf{B}|$ [18-20]. Ion scattering is expected around the strongest magnetic field gradient [21, 22], i.e. around the $B_{l}$ reversal.

We fit the observed magnetic field around the discontinuity center $\left(B_{l}\right.$ reversal) by a simple model: $B_{l} \approx B_{0}$. $\left(r_{n} / L\right), B_{n}=$ const, $B_{m}=\sqrt{B_{0}^{2}-B_{l}^{2}} \approx B_{0}\left(1-r_{n}^{2} / 2 L^{2}\right)$ where $L$ is the discontinuity thickness, $L \approx c B_{0} / 4 \pi j_{m} \approx$ $250 \mathrm{~km}$. The ion (of mass $m$ and charge $q$ ) motion in such a field is given by the Hamiltonian:

$$
\begin{aligned}
H & =\frac{1}{2 m} p_{n}^{2}+\frac{1}{2 m}\left(p_{l}-\frac{q B_{0}}{c}\left(r_{n}-\frac{r_{n}^{3}}{6 L^{2}}\right)\right)^{2} \\
& +\frac{1}{2 m}\left(p_{m}-\frac{q B_{n}}{c} r_{l}+\frac{q B_{0}}{c} \frac{r_{n}^{2}}{2 L}\right)^{2}
\end{aligned}
$$

where momenta $\mathbf{p}=\left(p_{l}, p_{m}, p_{n}\right)$ are conjugate to coordinates $\mathbf{r}=\left(r_{l}, r_{m}, r_{n}\right)$. We introduce dimensionless variables $(x, z)=\left(r_{l}-p_{m} c / q B_{n}, r_{n}\right) / \sqrt{L \rho},\left(p_{x}, p_{z}\right)=$ $\left(p_{l}, p_{n}\right) / \sqrt{h m}$ (note $p_{m}=$ const because $\partial H / \partial r_{m}=0$ ), $H \rightarrow H / h, \kappa=\left(B_{n} / B_{0}\right) \sqrt{L / \rho}$, where $\rho=\sqrt{2 h m} c / q B_{0}$ and $h=\left(q B_{0} L / m c\right)^{2} m$ (i.e., $\rho=L$ for this normalization), and rewrite Eq. (1) as:

$$
H=\frac{1}{2} p_{z}^{2}+\frac{1}{2}\left(p_{x}-z+\frac{z^{3}}{6}\right)^{2}+\frac{1}{2}\left(\kappa x-\frac{z^{2}}{2}\right)^{2}
$$

The distinctive feature of Hamiltonian in Eq. (2) is the term $\sim z^{3} / 6$ describing magnetic field $B_{m}$ peak; previous analysis of ion motion was limited to Hamiltonians with $p_{x}^{2} / 2[22,28]$ or $\left(p_{x}-z\right)^{2} / 2[29,30]$ terms, describing discontinuities with $B_{m}=0$ and $B_{m}=$ const. In this study we demonstrate that the new term $\sim z^{3} / 6$ qualitatively changes the ion scattering efficiency. 

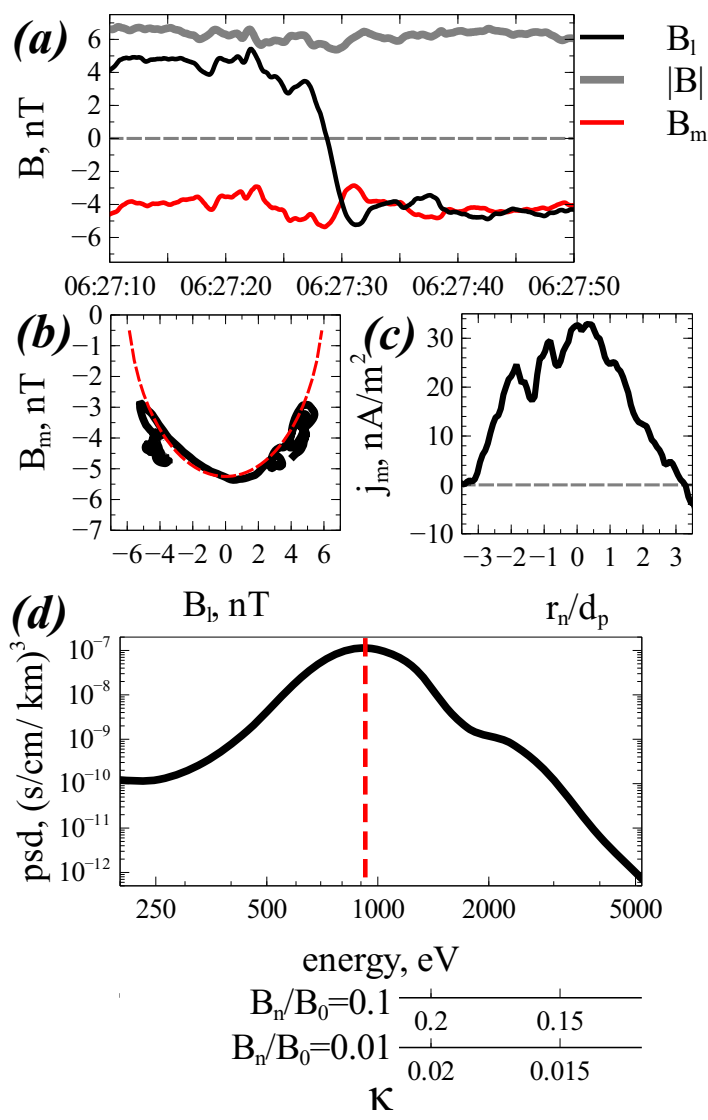

FIG. 1. Example of a discontinuity observed by ARTEMIS [17] on 2020-02-19: (a) magnetic field in the discontinuity coordinate system ( $\mathbf{l}$ is the maximum variance direction, $\mathbf{m}$ is the intermediate variance direction), (b) magnetic field hodogram $\left(B_{l}, B_{m}\right)$ and the circle $\left(B_{m}-0.75 \mathrm{nT}\right)^{2}+B_{l}^{2} \approx$ $36 \mathrm{nT}^{2}$ (red dased curve), (c) current density profile, (d) ion energy spectrum with the solar wind flow energy $m_{p} v_{s w}^{2} / 2$ shown with red dashed line; two bottom axes show $\kappa$ parameter calculated for the spectrum energy minus the flow energy, $\Delta E=E-m_{p} v_{s w}^{2} / 2(\Delta E$ is zero for ions moving with the solar wind; two $B_{n}$ values are used for $\kappa$ ). We show $\kappa$ for the energetic part of spectrum only as interaction of these ions with SWD is considered. Magnetic field at $0.25 \mathrm{~s}$ resolution is from the fluxgate magnetometer [23], whereas ions at $4 \mathrm{~s}$ resolution, the spin-period, is from the electrostatic analyzer [24]. The local coordinate system and the current density are obtained from maximum variance analysis [25] and timing, respectively [see details in 18]. ARTEMIS data are processed with SPEDAS V3.1 [26]. Two ARTEMIS observations are not sufficient to accurately estimate the direction along the normal $\mathbf{n}$ to the SWD surface [see discussion in 27], and thus we use a range of $B_{n}$ values (normalized to the $B_{l}$ magnitude) for the $\kappa$ calculation in panel $(\mathrm{d})$.

In most discontinuities the observed $B_{n} / B_{0} \ll 1$ [31], i.e. $\kappa \ll 1$ and variables $\left(\kappa x, p_{x}\right)$ change much slower than $\left(z, p_{z}\right)$ (see the range of $\kappa$ values in Fig. 1(d)). This determines the character of the ion interaction with discontinuities. For frozen $\left(\kappa x, p_{x}\right)$, the Hamiltonian (2) describes periodic motion in the $\left(z, p_{z}\right)$ plane with con- served generalized magnetic moment $I_{z}=(2 \pi)^{-1} \oint p_{z} d z$. For slow changes of $\left(\kappa x, p_{x}\right) I_{z}$ remains conserved, now as an adiabatic invariant [32]. $I_{z}$ only changes due to a significant change of the trajectory configuration [33]. The conservation of $I_{z}$ and energy (Hamiltonian in Eq. (2) is conservative) is sufficient to fully integrate the ion motion, and thus without $I_{z}$ destruction there is no ion scattering at discontinuities. Generally, the adiabatic invariant $I_{z}$ is conserved with an exponential accuracy $\sim \exp (-$ const $/ \kappa)[21,34,35]$, i.e. ion scattering is quite weak (slow). However, the Hamiltonian in Eq. (2) describes two different types of ion motions in the $\left(z, p_{z}\right)$ plane (see Fig. 2(a) showing two types of phase portraits), and change of one type of motion to another type corresponds to an $I_{z}$ jump of the order of $\sim \kappa$ or $\sim 1$ due to crossing of the separatrix demarcating phase domains with two types of motions [36-38].

Jumps of $I_{z}$ due to separatrix crossings have been studied for special cases of the Hamiltonian of Eq. (2) with $\sim p_{x}^{2} / 2$ [22] and $\sim\left(p_{x}-z\right)^{2} / 2$ terms [30]. In both systems these jumps are found to be random with an amplitude $\sim \kappa$ and zero mean value, $\left\langle\Delta I_{z}\right\rangle=0$ after averaging over many separatrix crossings, i.e. there is slow diffusion of $I_{z}$ with variance $\left\langle\left(\Delta I_{z}\right)^{2}\right\rangle \sim \kappa^{2}$ and diffusion rate $\left\langle\left(\Delta I_{z}\right)^{2}\right\rangle / \tau \sim \kappa^{3} \ll 1[39]$ where $\tau \sim 1 / \kappa$ is the timescale between two separatrix crossings (time-scale of the ion motion in the $\left(\kappa x, p_{x}\right)$ plane).

Besides the exponential $\sim \exp (-$ const $/ \kappa)$ or slow $\sim \kappa^{3}$ destruction of $I_{z}$ for systems with $\kappa \ll 1$, there also exists a so-called geometrical destruction (with $\left\langle\Delta I_{z}\right\rangle \sim 1$ ) predicted theoretically [40] and not yet found for conservative Hamiltonians describing ion interaction with discontinuities [see discussion in 30]. In this study we show that the term $\sim z^{3} / 6$ (i.e., $B_{m}$ peak) results in such geometrical $I_{z}$ destruction and produces very fast ion scattering.

To show the effect of $I_{z}$ change due to separatrix crossings, we color the $\left(\kappa x, p_{x}\right)$ plane according to the phase portraits in a $\left(z, p_{z}\right)$ plane. Note the two types of phase portraits correspond to two profiles of effective potential energy $U(z)=H-p_{z}^{2} / 2$ : there are two potential wells (one or two possible orbits in the $\left(z, p_{z}\right)$ plane at fixed $H)$ in the left portrait in Fig. 2(a), and there is a single potential well (one possible orbit in the $\left(z, p_{z}\right)$ plane at fixed $H$ ) in the right portrait shown in Fig. 2(a). In Figure 2(c,d) the region with a single possible orbit is yellow and the region with two possible orbits is red (lines $\ell_{1-5}$ demarcate these regions). Orbits crossing $\ell_{1-4}$ do not change their potential well of $U(z)$, i.e. the corresponding particles continue oscillating in the same well. Separatrix crossings occur when orbits cross (or reflect from) the boundary $\ell_{5}$. Particles moving with $\kappa x$ decreasing at crossing $\ell_{5}$ change oscillations in one of the two potential wells to oscillations covering the both wells (and vise versa for particles moving with $\kappa x$ increase). Particles moving with $\kappa x$ decrease and reflecting from $\ell_{5}$ 

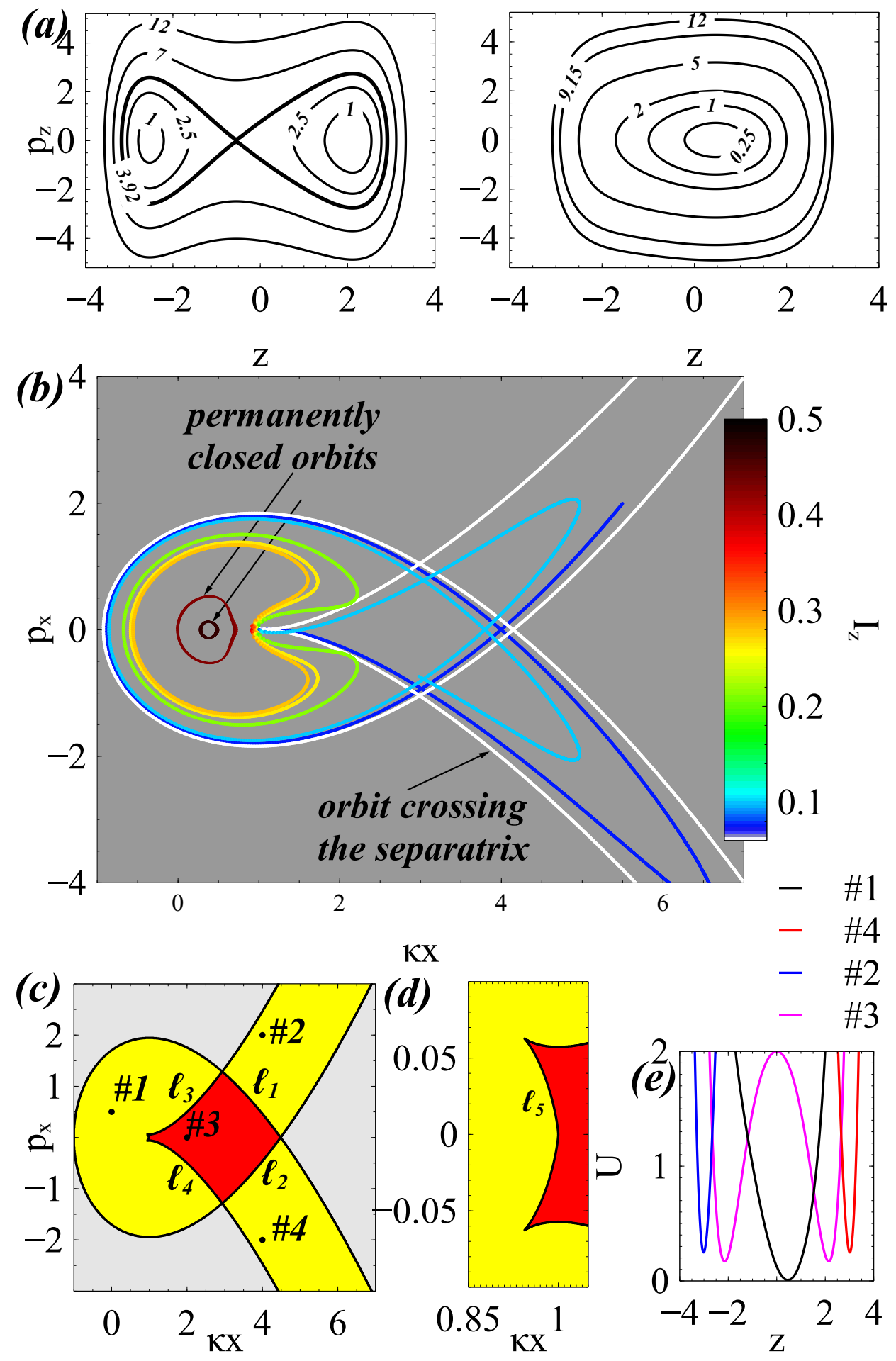

FIG. 2. Properties of Hamiltonian system of Eq. (2) for total energy $H=1 / 2$. (a) Phase portraits of Hamiltonian (2) at frozen $\left(\kappa x, p_{x}\right): H$ values of each curve are shown in the plot; $\kappa x=2.5, p_{x}=1$ in the left panel and $\kappa x=0, p_{x}=0.5$ in the right panel. (b) Ion trajectories for many periods of slow motion in the $\left(\kappa x, p_{x}\right)$ plane $(\kappa=0.01)$; color shows instantaneous value of $I_{z}$; arrows show different types of orbits: except for the permanently closed orbits that never cross the separatrix, all other curves are sections of the same orbit reflecting from $\ell_{5}$ and changing $I_{z}$ at these moments. All the orbits have $H=1 / 2$ and initial $\left(\kappa x, p_{x}\right)=(5.5,2.0),(0.0,-3.5),(0.0,0.5)$. (c) Plane $\left(\kappa x, p_{x}\right)$ colored according to the type of motion in $\left(z, p_{z}\right)$ plane with frozen $\left(\kappa x, p_{x}\right)$ : yellow is for a single possible orbit in the $\left(z, p_{z}\right)$ plane at fixed $H=1 / 2$ (the right phase portrait in the panel (a) or the left phase portrait in the panel (a), but with only one orbit having $H=1 / 2$ ), red is for two possible orbits in the $\left(z, p_{z}\right)$ plane at fixed $H=1 / 2$ (the left phase portrait in the panel (a)), grey is for the absence of solutions. (d) Zoomed-in section of (c) at $\kappa x \in[0.85,1.05]$ and $p_{x} \in[-0.1,0.1]$. (e) $1 \mathrm{D}$ profiles of potential energy $U(z)=H-p_{z}^{2} / 2$ at frozen $\left(\kappa x, p_{x}\right)$ : numbers correspond to \# marks in (c). 
change oscillations in one of the two potential wells to oscillations in another well. Such crossings correspond to change of the well that results in $I_{z}$ jump (this $2 \pi \Delta I_{z}$ jump equals to the difference of areas of two regions surrounded by the separatrix in the $\left(z, p_{z}\right)$ plane and does not depend on $\kappa$, see [33]). This is why $I_{z}$ always changes when the orbit reflects from $\ell_{5}$ in $\left(\kappa x, p_{x}\right)$ plane (and thus switches from oscillations in one well to oscillations in another well in $\left(z, p_{z}\right)$ plane): Fig. 2(b) demonstrates the change of $I_{z}$ (shown in color) for a single orbit.

There are two main populations of trajectories in the $\left(\kappa x, p_{x}\right)$ plane. The first consists of trajectories reflected from $\ell_{5}$ curve and quickly scattered due to $\Delta I_{z} \sim 1$ jumps. The second consists of so-called closed trajectories trapped within the discontinuity, which never arrive close to $\ell_{5}$ (see Fig. 2(b)). Exchange between these two ion populations should be controlled by external forces and dissipative processes (e.g., wave-particle interactions [41] or discontinuity evolution [10, 42]). Such evolution and (or) dissipation makes the system non-conservative (i.e., particle energy changes during particle interaction with the discontinuity), and results in evolution of the boundary separating these two populations in the phase space $[39,43]$. A third (minor) population of quasi-closed trajectories that cross $\ell_{5}$ rarely, is quite small for Hamiltonian (2). Let us discuss peculiarities of these three populations regarding their interaction with $\ell_{5}$.

The first two populations are: (i) regular trajectories never approaching $\ell_{5}$ with $I_{z} \approx$ const (in the $\left(\kappa x, p_{x}\right)$ plane these trajectories occupy region located to the left from $\ell_{5}$ ); (ii) transient trajectories reflecting from $\ell_{5}$. Due to geometrical $I_{z}$ jumps trajectories of this latter population are characterized by rapid destruction of $I_{z}$ invariant. The population (iii) consists of ion trajectories that cross $\ell_{5}$. We can call these trajectories quasiregular, because ions on these trajectories can spend a long time on the boundary of the regular trajectory region $\left(I_{z} \approx\right.$ const for this motion), but rarely such a trajectory crosses $\ell_{5}$ and goes to the $\left(\kappa x, p_{x}\right)$ region of transient trajectories. Figure 3 shows an example of this quasi-regular behavior: the ion moves along a quasiclosed orbit on the left from $\ell_{5}$ (within the region of closed trajectories) for quite a long period of time; then finally the weak scattering in $I_{z}$ results in crossing $\ell_{5}$ and escape into the region of transient trajectories. An analog of this orbit with the ion moving in the opposite direction would be represented by the ion approaching $\ell_{5}$ from the right and then crossing $\ell_{5}$. Note that the absolute majority of trajectories approaching $\ell_{5}$ from the right are transient trajectories, which are reflected from $\ell_{5}$.

To estimate the amount of such quasi-regular trajectories we use the Poincaré section technique: we run $10^{5}$ trajectories with initial 10 values of $\kappa x \in[-1,1], p_{x}=0$ and uniformly distributed $z$; then we plot the points where the trajectories cross $z=0$ plane with $p_{z}>0$. These points are spread within the circle $(\kappa x)^{2}+p_{x}^{2}=2 H$

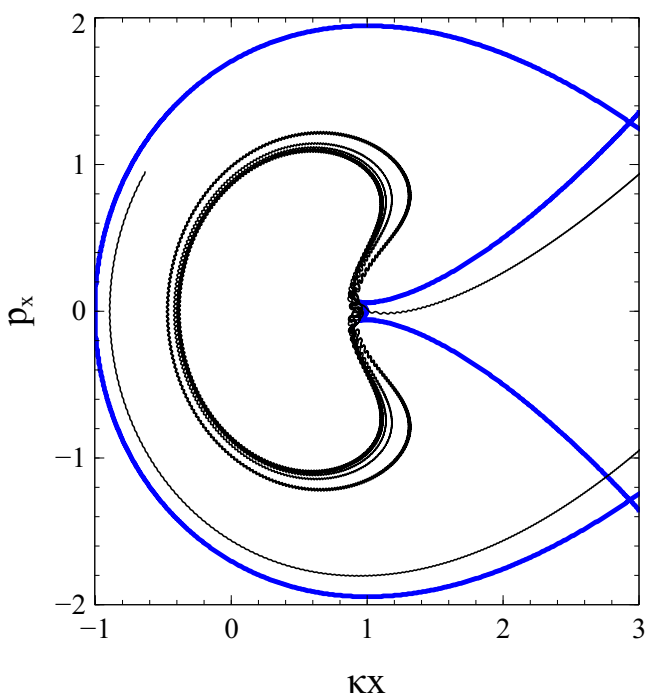

FIG. 3. Quasi-regular trajectory (black curve) of the ion moving along a quasi-closed orbit within the region of regular trajectories and then escaping this region by crossing $\ell_{5}$. Blue shows the boundary of the region of allowed particle motion (outer curve) and $\ell_{1}-\ell_{5}$ curves.

and their distribution shows the three populations of trajectories (see Fig. 4): (i) ions moving along regular trajectories fill closed curves in the Poincaré section; (ii) quickly scattered ions on transient trajectories fill some domain in the Poincaré section by random and sparse points (note the density of points is determined by the typical time-scale between two successive crossings of $z=0$ plane, and rapidly scattered ions can get quite small $I_{z}$ corresponding to strongly elongated trajectories in the $\left(\kappa x, p_{x}\right)$ plane with long excursions between two $z=0$ crossings); (iii) ions moving along quasi-regular trajectories cross $z=0$ with a short period (regular trajectories correspond to large $I_{z}$ ), but positions of crossings are randomly distributed. Figure 4 demonstrates that quasi-regular trajectories (crossing $\ell_{5}$ ) fill a layer separating regular trajectories and transient trajectories, but the total area of these quasi-regular trajectories is smaller than the areas filled by both regular and transient trajectories.

Figure 2(b) shows that different sections of orbit crossing the separatrix are associated to quite different $I_{z}$ values. This is an important property of the geometrical $I_{z}$ destruction: jumps $\left\langle\Delta I_{z}\right\rangle \sim 1$ do not depend on $\kappa$ and are quite large, i.e. even for arbitrarily small $\kappa$ the ion scattering is fast and effective. Note $\kappa \rightarrow 0$ means the transition between rotational (with $B_{n} \neq 0$ ) and tangential $\left(B_{n} \rightarrow 0\right)$ types of SWDs [44]. Figure 5 (left panel) shows evolution of initially narrow $I_{z}$-distribution for two $\kappa$ values: geometrical jumps of $I_{z}$ quickly widen $I_{z}$-distributions. The scattering rate in the slow time $\kappa t$ does not depend on $\kappa$. However, the rotation period $\tau$ of particle motion in the $\left(\kappa x, p_{x}\right)$ plane is of or- 


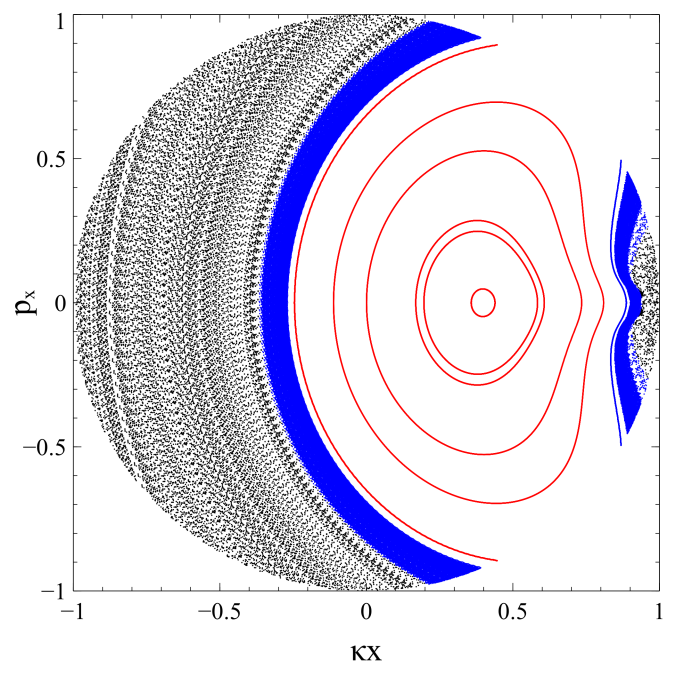

FIG. 4. Poincaré section of the system (2): the closed red curves show particles moving along regular trajectories; black random sparse dots show particles moving along transient trajectories; blue random dense dots show particles moving along quasi-regular trajectories, which cross $\ell_{5}$.

der $1 / \kappa$. This period determines the time scale of the ion interaction with the same SWD, i.e. after one separatrix crossing ions turn around at large $\kappa x$ and come back to the separatrix again after $\sim \tau$ period. Ion scattering and destruction of $I_{z}$ is equivalent to the pitchangle scattering, because $I_{z}$ determines the ion pitchangle, e.g. at $z=0, p_{x}=0$ the ion pitch-angle is defined as $\cos \alpha=\mathbf{v} \cdot \mathbf{B} / B v=\left(\kappa x+\sqrt{2 h-(\kappa x)^{2}}\right) / \sqrt{1+\kappa^{2}}$. Each trajectory in $\left(\kappa x, p_{x}\right)$ plane is associated with some $I_{z}$ value, and jump of $I_{z}$ means change of trajectory. As each trajectory is characterized by some $\kappa x$ at $p_{x}=0$ crossing, jumps of $I_{z}$ result in changes of this $\kappa x$ (see Fig. 2(b)). Thus, $I_{z}$ jumps lead to values of $\kappa x$ at $p_{x}=0$ jumps and to $\alpha=\alpha(\kappa x)$ jumps (note $h=$ const for ion scattering). Figure 5 (right panel) shows evolution of pitch-angle distributions recalculated from $I_{z^{-}}$ distributions: starting with a narrow $\alpha$-distribution, ions are quickly scattered and fill a broad $\alpha$ range.

To demonstrate the effect of magnetic field component $B_{m}$ on ion scattering we compare $I_{z}, \alpha$-distributions obtained for Hamiltonian (2) with $\left(p_{x}-z+z^{3} / 6\right)^{2}$ term $\left(B_{m}\right.$ peak $)$ and with $p_{x}^{2} / 2$ term $\left(B_{m}=0\right.$; classical compressional discontinuity, see [22]). Figure 5 shows that in the absence of $B_{m}$ peak (i.e., when $B_{m}=0$ ) the ion scattering is quite weak: $I_{z}, \alpha$-distributions are narrow peaks around the initial distribution (in agreement with theoretical predictions of slow $I_{z}$ destruction, see $[36,37]$ ).

Although Fig. 1 shows almost force-free discontinuity ( $B \approx$ const), the $B_{m}$ magnitude is slightly smaller than $B_{l}$ magnitude, i.e. the field model $B_{m}^{2}+B_{l}^{2}=$ const in Hamiltonian (2) is an approximation. To study ion interaction with discontinuities having $B_{m} / B_{0}<1$ we consider statistics of ion-scale $L \sim d_{i}$ discontinuities from
[18]. Figure 6 (a) shows $B_{m} / B_{0}$ distribution and $\Delta B /\langle B\rangle$ (deviation from the force-free condition) for $\sim 250$ individual discontinuities: there is a significant fraction of $B_{m} / B_{0} \in[0.5,0.75]$ events, whereas for most of events $\Delta B / B \sim 0.03$. To examine how effect of strong $I_{z}$ destruction depends on $B_{m} / B_{0}$ we plot $I_{z}$-distributions for $\kappa t=100$ simulation time and different $B_{m} / B_{0}$ values in Hamiltonian (2). For $B_{m} / B_{0}>0.75$ the $I_{z}$ destruction rate is comparable to one for $B_{m} / B_{0}=1$, whereas for $B_{m} / B_{0}<0.75$ the $I_{z}$ destruction rate is smaller and resulted $I_{z}$-distribution is narrower. Therefore, the effect of the fast $I_{z}$ destruction remains actual for a wide range of observed $B_{m} / B_{0}$ values. Note we consider rotational discontinuities that are typically Alfvenic-type structures with $B \approx$ const $[10,18,31]$, but solar wind ions also interact with various compressional structures contributing to the solar wind magnetic field spectrum [45, 46], e.g. mirror mode structures [e.g., 47, 48] and interplanetary shocks [e.g., 49-51]. Investigation of ion scattering by such structures (with $B_{m} / B_{0} \ll 1$ and asymmetrical $B_{l}$ profiles) deserves a separate study. An important element of compressional structures with the ion kinetic scales is a polarization electric field forming due to the decoupling of ion and electron motions [e.g., $52]$. Such field almost absents in the force-free discontinuities [53], but can strongly influence ion scattering in compressional discontinuities [54]. Therefore, further investigations of ion interaction with partially force-free and compressional structures would require inclusion polarization fields into consideration.

In Hamiltonian (2), $\kappa$ determines the time interval between two scatterings (a time scale of one period of ion motion in the $\left(\kappa x, p_{x}\right)$ plane). In systems with multiple SWDs, the time-scale between scatterings would be partially controlled by the SWD occurrence rate, which does not depend on $\kappa$. This further reduces the importance of $B_{n}$ (or $\left.\kappa\right)$ value for the ion scattering, and makes separation between almost tangential (small, but finite $B_{n}$ ) and rotational SWDs unimportant for determination of their role in ion scattering. Thus, we show that $B_{n}$ amplitude ( $\kappa$ value) does not control the ion scattering rate in the presence of geometrical $I_{z}$ destruction, i.e. ions are similarly scattered by rotational and almost tangential (with small, but finite $B_{n}$ ) SWDs.

This scattering is a universal mechanism for SWDs with the $B_{m}$ peak comparable to the $B_{l}$ magnitude (the most common configuration of compressionless SWDs, e.g. $[1,10,19,42]$ ) and sufficiently hot ions (or sufficiently thin SWDs; $L \sim \rho$ ). The condition $L \sim \rho$ can be rewritten for the ion energy $h / T_{i} \sim \beta_{i}\left(L / d_{p}\right)^{2}$ with $T_{i}$ and $\beta_{i}$ being solar wind temperature and the ratio of thermal and magnetic pressures. For most intense discontinuities $L / d_{p} \in[1,10]$ [18-20], and typical $\beta_{i} \in[0.1,10][$ see 55]. Thus, almost all suprathermal solar wind population can be strongly scattered due to interaction with SWDs. This effect, together with scattering 

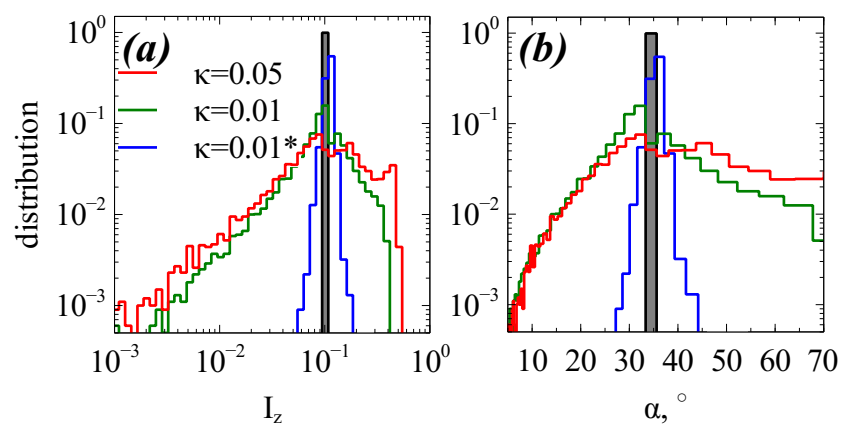

FIG. 5. Panel (a) shows $I_{z}$-distributions and panel (b) shows pitch-angle distributions (we use pitch-angle values evaluated at the $z=0$ plane): (red) $\kappa=0.05$, (green) $\kappa=0.01$, (blue) $\kappa=0.01$ and Hamiltonian (2) contains $p_{x}^{2} / 2$ instead of $\left(p_{x}-z+z^{3} / 6\right)^{2} / 2$. The initial distribution is shown by grey, whereas all colored distributions correspond to $\kappa t=100$ simulation time.
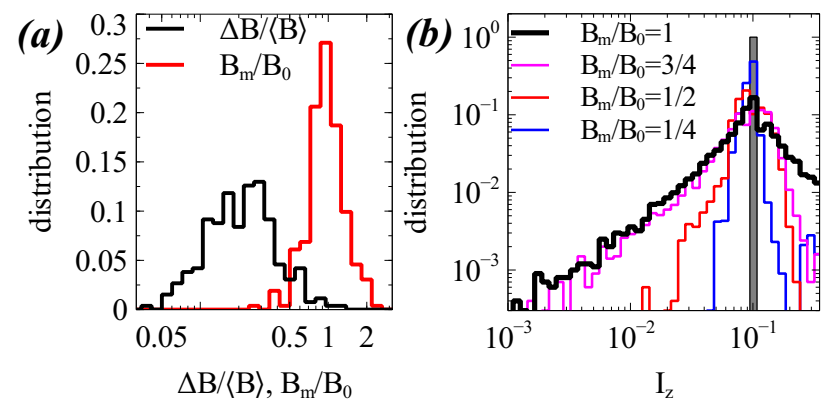

FIG. 6. Panel (a) shows distribution of characteristics of ion-scale discontinuities from [18] statistics: compressionality $\Delta B /\langle B\rangle$ (with $\Delta B=\max B-\min B$ ) and $B_{m} / B_{0}$. Panel (b) shows $I_{z}$-distributions for $\kappa=0.01$ and different $B_{m} / B_{0}$. Grey color shows the initial $I_{z}$-distribution.

by plasma waves [e.g., 41, 56], should shape the observed low-anisotropic distribution of solar wind ions at $1 \mathrm{AU}$ [55] and contribute to the quick cross-field transport of high-energy ion populations [12-14].

We acknowledge the support NASA contract NAS502099 for ARTEMIS data available at http://themis. ssl.berkeley.edu/. Work of A.V.A., A.I.N., A.A.V., and A.A.V. was supported by the Russian Scientific Foundation, project 19-12-00313. A.I.N. also acknowledge support by the Leverhulme Thrust (grant \# RPG2018-143). Work of L.M.Z. was supported by the Russian Science Foundation (grant \# 20-42-04418).

* aartemyev@igpp.ucla.edu

[1] B. T. Tsurutani and C. M. Ho, Reviews of Geophysics 37, 517 (1999).

[2] R. Bruno and V. Carbone, Living Reviews in Solar Physics 2, 4 (2005).

[3] T. D. Phan, S. D. Bale, J. P. Eastwood, B. Lavraud,
J. F. Drake, M. Oieroset, M. A. Shay, M. Pulupa, M. Stevens, R. J. MacDowall, A. W. Case, D. Larson, J. Kasper, P. Whittlesey, A. Szabo, K. E. Korreck, J. W. Bonnell, T. D. de Wit, K. Goetz, P. R. Harvey, T. S. Horbury, R. Livi, D. Malaspina, K. Paulson, N. E. Raouafi, and M. Velli, Astrophys. J. Sup. 246, 34 (2020), arXiv:2001.06048 [astro-ph.SR].

[4] T. Dudok de Wit, V. V. Krasnoselskikh, S. D. Bale, J. W. Bonnell, T. A. Bowen, C. H. K. Chen, C. Froment, K. Goetz, P. R. Harvey, V. K. Jagarlamudi, A. Larosa, R. J. MacDowall, D. M. Malaspina, W. H. Matthaeus, M. Pulupa, M. Velli, and P. L. Whittlesey, Astrophys. J. Sup. 246, 39 (2020), arXiv:1912.02856 [astro-ph.SR].

[5] J. A. Tessein, W. H. Matthaeus, M. Wan, K. T. Osman, D. Ruffolo, and J. Giacalone, Astrophys. J. Lett. 776, L8 (2013).

[6] J. T. Gosling, Space Sci. Rev. 172, 187 (2012).

[7] W. H. Matthaeus, G. Qin, J. W. Bieber, and G. P. Zank, Astrophys. J. Lett. 590, L53 (2003).

[8] G. Zimbardo, P. Pommois, and P. Veltri, Astrophys. J. Lett. 639, L91 (2006).

[9] J. R. Jokipii, Astrophys. J. 146, 480 (1966).

[10] B. J. Vasquez, V. I. Abramenko, D. K. Haggerty, and C. W. Smith, Journal of Geophysical Research (Space Physics) 112, A11102 (2007).

[11] J. E. Borovsky, Physical Review Letters 105, 111102 (2010).

[12] J. Giacalone and J. R. Jokipii, Astrophys. J. 520, 204 (1999)

[13] C. Pei, J. R. Jokipii, and J. Giacalone, Astrophys. J. 641, 1222 (2006).

[14] J. Giacalone, J. R. Jokipii, and J. E. Mazur, Astrophys. J. Lett. 532, L75 (2000).

[15] P. Sun, J. R. Jokipii, and J. Giacalone, Astrophys. J. 827, 16 (2016).

[16] W. Horton, Physic. Rep. 192, 1 (1990).

[17] V. Angelopoulos, Space Sci. Rev. 165, 3 (2011).

[18] A. V. Artemyev, V. Angelopoulos, and I. Y. Vasko, Journal of Geophysical Research (Space Physics) 124, 3858 (2019).

[19] J. J. Podesta, J. Geophys. Res. 122, 2795 (2017).

[20] A. Greco, S. Perri, S. Servidio, E. Yordanova, and P. Veltri, Astrophys. J. Lett. 823, L39 (2016), arXiv:1511.03084 [astro-ph.SR].

[21] B. V. Chirikov, Reviews of Plasma Physics, 1st ed., Vol. 13 (Consultants Bureau, New York, 1987) pp. 1-92.

[22] J. Büchner and L. M. Zelenyi, J. Geophys. Res. 94, 11821 (1989).

[23] H. U. Auster, K. H. Glassmeier, W. Magnes, O. Aydogar, W. Baumjohann, D. Constantinescu, D. Fischer, K. H. Fornacon, E. Georgescu, P. Harvey, O. Hillenmaier, R. Kroth, M. Ludlam, Y. Narita, R. Nakamura, K. Okrafka, F. Plaschke, I. Richter, H. Schwarzl, B. Stoll, A. Valavanoglou, and M. Wiedemann, Space Sci. Rev. 141, 235 (2008).

[24] J. P. McFadden, C. W. Carlson, D. Larson, M. Ludlam, R. Abiad, B. Elliott, P. Turin, M. Marckwordt, and V. Angelopoulos, Space Sci. Rev. 141, 277 (2008).

[25] B. U. Ö. Sonnerup and L. J. Cahill, Jr., J. Geophys. Res. 73, 1757 (1968).

[26] V. Angelopoulos, P. Cruce, A. Drozdov, E. W. Grimes, N. Hatzigeorgiu, D. A. King, D. Larson, J. W. Lewis, J. M. McTiernan, D. A. Roberts, C. L. Russell, T. Hori, 
Y. Kasahara, A. Kumamoto, A. Matsuoka, Y. Miyashita, Y. Miyoshi, I. Shinohara, M. Teramoto, J. B. Faden, A. J. Halford, M. McCarthy, R. M. Millan, J. G. Sample, D. M. Smith, L. A. Woodger, A. Masson, A. A. Narock, K. Asamura, T. F. Chang, C.-Y. Chiang, Y. Kazama, K. Keika, S. Matsuda, T. Segawa, K. Seki, M. Shoji, S. W. Y. Tam, N. Umemura, B.-J. Wang, S.-Y. Wang, R. Redmon, J. V. Rodriguez, H. J. Singer, J. Vandegriff, S. Abe, M. Nose, A. Shinbori, Y.-M. Tanaka, S. UeNo, L. Andersson, P. Dunn, C. Fowler, J. S. Halekas, T. Hara, Y. Harada, C. O. Lee, R. Lillis, D. L. Mitchell, M. R. Argall, K. Bromund, J. L. Burch, I. J. Cohen, M. Galloy, B. Giles, A. N. Jaynes, O. Le Contel, M. Oka, T. D. Phan, B. M. Walsh, J. Westlake, F. D. Wilder, S. D. Bale, R. Livi, M. Pulupa, P. Whittlesey, A. DeWolfe, B. Harter, E. Lucas, U. Auster, J. W. Bonnell, C. M. Cully, E. Donovan, R. E. Ergun, H. U. Frey, B. Jackel, A. Keiling, H. Korth, J. P. McFadden, Y. Nishimura, F. Plaschke, P. Robert, D. L. Turner, J. M. Weygand, R. M. Candey, R. C. Johnson, T. Kovalick, M. H. Liu, R. E. McGuire, A. Breneman, K. Kersten, and P. Schroeder, Space Sci. Rev. 215, 9 (2019).

[27] T. Knetter, F. M. Neubauer, T. Horbury, and A. Balogh, J. Geophys. Res. 109, A06102 (2004).

[28] A. Neishtadt, C. Simo, D. Treschev, and A. Vasiliev, Discrete Contin. Dyn. Syst. Ser. B (2008).

[29] H. Karimabadi, P. L. Pritchett, and F. V. Coroniti, J. Geophys. Res. 95, 17153 (1990).

[30] A. V. Artemyev, A. I. Neishtadt, and L. M. Zelenyi, Phys. Rev. E 89, 060902 (2014).

[31] M. Neugebauer, J. Geophys. Res. 111, A04103 (2006).

[32] L. D. Landau and E. M. Lifshitz, Vol. 1: Mechanics, Course of Theoretical Physics (Oxford: Pergamon, 1988).

[33] V. I. Arnold, V. V. Kozlov, and A. I. Neishtadt, Mathematical Aspects of Classical and Celestial Mechanics, 3rd ed., Dynamical Systems III. Encyclopedia of Mathematical Sciences (Springer-Verlag, New York, 2006).

[34] T. J. Birmingham, J. Geophys. Res. 89, 2699 (1984).

[35] A. I. Neishtadt, Regular and chaotic dynamics 5, 213 (2000).

[36] A. I. Neishtadt, Soviet Journal of Plasma Physics 12, 568 (1986).

[37] J. R. Cary, D. F. Escande, and J. L. Tennyson, Physical Review A 34, 4256 (1986).

[38] J. L. Tennyson, J. R. Cary, and D. F. Escande, Physical Review Letters 56, 2117 (1986).

[39] L. M. Zelenyi, A. I. Neishtadt, A. V. Artemyev, D. L. Vainchtein, and H. V. Malova, Physics Uspekhi 56, 347 (2013).

[40] A. Neishtadt and D. Treschev, Ergodic theory and dynamical systems 31, 259 (2011).
[41] P. Hellinger, P. Trávníček, J. C. Kasper, and A. J. Lazarus, Geophys. Res. Lett. 33, L09101 (2006).

[42] A. Tenerani and M. Velli, Astrophys. J. Lett. 867, L26 (2018), arXiv:1808.04453 [physics.plasm-ph].

[43] D. L. Vainchtein, J. Büchner, A. I. Neishtadt, and L. M. Zelenyi, Nonlinear Processes in Geophysics 12, 101 (2005).

[44] P. D. Hudson, Plan. Sp. Sci. 18, 1611 (1970).

[45] P. Veltri and A. Mangeney, in American Institute of Physics Conference Series, American Institute of Physics Conference Series, Vol. 471, edited by S. R. Habbal, R. Esser, J. V. Hollweg, and P. A. Isenberg (1999) pp. 543-546.

[46] P. Veltri, G. Nigro, F. Malara, V. Carbone, and A. Mangeney, Nonlinear Processes in Geophysics 12, 245 (2005).

[47] C. T. Russell, L. K. Jian, J. G. Luhmann, T. L. Zhang, F. M. Neubauer, R. M. Skoug, X. Blanco-Cano, N. Omidi, and M. M. Cowee, Geophys. Res. Lett. 35, L15101 (2008).

[48] M. Volwerk, C. Goetz, F. Plaschke, T. Karlsson, and D. Heyner, Annales Geophysicae Discussions 2019, 1 (2019).

[49] L. B. Wilson, III, A. Koval, A. Szabo, A. Breneman, C. A. Cattell, K. Goetz, P. J. Kellogg, K. Kersten, J. C. Kasper, B. A. Maruca, and M. Pulupa, Geophys. Res. Lett. 39, L08109 (2012).

[50] E. L. M. Hanson, O. V. Agapitov, F. S. Mozer, V. Krasnoselskikh, S. D. Bale, L. Avanov, Y. Khotyaintsev, and B. Giles, Geophys. Res. Lett. 46, 2381 (2019).

[51] I. J. Cohen, S. J. Schwartz, K. A. Goodrich, N. Ahmadi, R. E. Ergun, S. A. Fuselier, M. I. Desai, E. R. Christian, D. J. McComas, G. P. Zank, J. R. Shuster, S. K. Vines, B. H. Mauk, R. B. Decker, B. J. Anderson, J. H. Westlake, O. Le Contel, H. Breuillard, B. L. Giles, R. B. Torbert, and J. L. Burch, Journal of Geophysical Research (Space Physics) 124, 3961 (2019).

[52] K. Schindler, J. Birn, and M. Hesse, Physics of Plasmas 19, 082904 (2012).

[53] T. Neukirch, I. Y. Vasko, A. V. Artemyev, and O. Allanson, Astrophys. J. 891, 86 (2020), arXiv:2001.11380 [physics.space-ph].

[54] E. Tsai, A. V. Artemyev, and V. Angelopoulos, Physics of Plasmas 24, 012908 (2017), arXiv:1612.06944 [physics.plasm-ph].

[55] L. B. Wilson, III, M. L. Stevens, J. C. Kasper, K. G. Klein, B. A. Maruca, S. D. Bale, T. A. Bowen, M. P. Pulupa, and C. S. Salem, Astrophys. J. Sup. 236, 41 (2018), arXiv:1802.08585 [physics.plasm-ph].

[56] P. H. Yoon, J. Seough, C. S. Salem, and K. G. Klein, Phys. Rev. Lett. 123, 145101 (2019). 